\title{
Remote Sensing and Modeling of Atmospheric Dust and Studying Its Impact on Environment, Weather, and Climate
}

\author{
Hesham El-Askary, ${ }^{1,2,3}$ Seon K. Park, ${ }^{4,5,6,7}$ Slobodan Nickovic, ${ }^{8,9,10}$ and Mian Chin ${ }^{11}$ \\ ${ }^{1}$ Schmid College of Science and Technology, Chapman University, Orange, CA 92866, USA \\ ${ }^{2}$ Center of Excellence in Earth Observing, Chapman University, Orange, CA 92866, USA \\ ${ }^{3}$ Department of Environmental Sciences, Alexandria University, Moharam Bek, Alexandria 21522, Egypt \\ ${ }^{4}$ Department of Environmental Science and Engineering, Ewha Womans University, Seoul 120-750, Republic of Korea \\ ${ }^{5}$ Department of Atmospheric Science and Engineering, Ewha Womans University, Seoul 120-750, Republic of Korea \\ ${ }^{6}$ Severe Storm Research Center, Ewha Womans University, Seoul 120-750, Republic of Korea \\ ${ }^{7}$ Center for Climate/Environment Change Prediction Research, Ewha Womans University, Seoul 120-750, Republic of Korea \\ ${ }^{8}$ Institute for Atmospheric Physics, The University of Arizona, Tucson, AZ 85721, USA \\ ${ }^{9}$ South East European Virtual Climate Change Center, Republic Hydrometeorological Service, Kneza Viseslava 66, \\ 11000 Belgrade, Serbia \\ ${ }^{10}$ Institute of Physics, 11080 Belgrade, Serbia \\ ${ }^{11}$ Atmospheric Chemistry and Dynamics Laboratory, NASA Goddard Space Flight Center, Code 614, Greenbelt, MD 20771, USA
}

Correspondence should be addressed to Hesham El-Askary; elaskary@chapman.edu

Received 23 June 2015; Accepted 23 June 2015

Copyright (c) 2015 Hesham El-Askary et al. This is an open access article distributed under the Creative Commons Attribution License, which permits unrestricted use, distribution, and reproduction in any medium, provided the original work is properly cited.

Aerosols have been regarded as one of the major constituents of the atmosphere and have a wide range of impacts at different levels, scales, and sectors. Remote sensing and modeling of the atmospheric dust storms, supported by ground observations, are critical in analyzing, characterizing, and assessing many impacts in terms of dust mineralogical composition, interaction with clouds and radiation, optical and chemical characteristics, major sources of emission, mixup with anthropogenic, sea-salt, and biomass aerosols during long range transport, and deposition. Studies of dust storms and their impact on the local and regional environment and climate, using space observations (Moderate Resolution Imaging Spectroradiometer (MODIS), Atmospheric Infrared Sounder (AIRS), Multiangle Imaging Spectroradiometer (MISR), Cloud-Aerosol Lidar and Infrared Pathfinder Satellite Observation (CALIPSO), Polarization and Anisotropy of Reflectances for Atmospheric Science coupled with Observations from a Lidar (PARASOL), Scanning Imaging Absorption spectrometer for Atmospheric Chartography (SCIAMACHY), Ozone Monitoring Instrument (OMI), etc.), ground measurements (Aerosol Robotic Network (AERONET), sky radiometers, sunphotometer, lidar systems, and chemical analysis), and modeling (SKIRON, Dust Regional Atmospheric Model (DREAM), Navy Aerosol Analysis and Prediction System (NAAPS), etc.), have addressed some of these issues. The studies included in this special issue are mainly concerned with ground-based, satellite observations and modeling of aerosol related events of natural or anthropogenic origin over climatically sensitive areas influenced by dust storms, such as Korea, China, and Saudi Arabia.

S. K. Park et al. investigated the temporal characteristics of major air pollutants collected from 44 air quality stations over the city of Seoul, Korea, namely, nitrogen dioxide, carbon monoxide, particular matter at 10 microns, and sulfur dioxide $\left(\mathrm{SO}_{2}\right)$, between 2000 and 2009. Ground data were correlated with satellite based observations collected from Moderate Resolution Imaging Spectroradiometer as well as the AERONET ground stations. They showed that anthropogenic aerosols are dominant in the summer season even though the concentration was lower than the other seasons which can be attributed to natural and anthropogenic sources, as well as changing meteorological factor. 
P. Li et al. studied the origin and distribution of polycyclic aromatic hydrocarbons (PAHs) originating from natural and/or anthropogenic sources in ambient particulate samples at high mountain region in southern China to understand their deposition and transport. Local meteorology including atmospheric pressure, relative humidity, and ambient temperature with backward trajectory analysis suggested that particulate samples measured at the Mount Heng region were predominantly associated with the air masses from southern China. They found out that vehicular emission, coal combustion, industry emission, and unburned fossil fuels were suggested to be the PAHs sources at Mount Heng site.

A. Farahat et al. covered aerosol distribution and characteristics using satellite based observations over four regions in Saudi Arabia using satellite and ground observations for the period April 2003-January 2013. The study includes cities in the north western, western, and eastern provinces of Saudi Arabia and in the Rub' al Khali desert or Empty Quarter. Satellite and ground observations showed that the dust season extends from April to August with prominent peaks yet with high anthropogenic contribution in late summer and early fall. Analysis shows an increase in the aerosol concentration during March 2009 which could be attributed to a major dust storm during that time.

J. Li and Z. Han shed the light on the three-dimensional evolution of an East Asian dust storm during 23-26 April 2009 by utilizing a Regional Air Quality Model System (RAQMS) and satellite measurements. Modeled spatial distributions of Aerosols Optical Depth (AOD) and vertical profiles of aerosol extinction coefficient during the dust storm were compared with MODIS and CALIPSO data, demonstrating that RAQMS was able to reproduce the $3 \mathrm{D}$ structure and the evolution of the dust storm reasonably well.

Y.-H. Jen et al. allocated sources of the long range transport of Asian dust across Taiwan using innovative chemicalassisted identification techniques. They combined chemical composition with the enrichment factor and the grey relational analysis to identify the potential sources of eighteen different Asian dust storms. Back trajectory analysis highlighted the source soils to be from Inner Mongolia with a high similarity with the outputs obtained from the chemical based techniques.

$\mathrm{H}$. Xu et al. studied dust aerosol properties near Hexi Corridor in the Chinese deserts using polarimetric measurements from a ground-based sunphotometer. They found out that the partial occurrence of calcite or hematite in the soil of Minqin or the influence of anthropogenic aerosols containing carbon affected aerosol characteristics as evident from the aerosol optical properties, angstrom exponent, and the single scattering albedo.

$\mathrm{H}$. Xu et al. used thermal observations of AIRS in conjunction with visible observations of MODIS and CloudAerosol Lidar with Orthogonal Polarization (CALIOP) for dust identification over the arid and semiarid regions of Asia. This is owed to the fact that dust generated in northern China exerts significant influences on regional air quality, weather, and climate.

J. Saydi et al. tackled the issue of increasing the laser ranging accuracy by ray tracing using atmospheric models based on surface measurements of pressure, temperature, and relative humidity. They studied the atmospheric effects on the laser beam by using the principles of laser ranging and performing atmospheric corrections for $0.532,1.3$, and 10.6 microns through the weather conditions of Tehran, Isfahan, and Bushehr in Iran. They found that the laser ranging error decreased by increasing the laser emission angle.

Hesham El-Askary Seon K. Park Slobodan Nickovic Mian Chin 

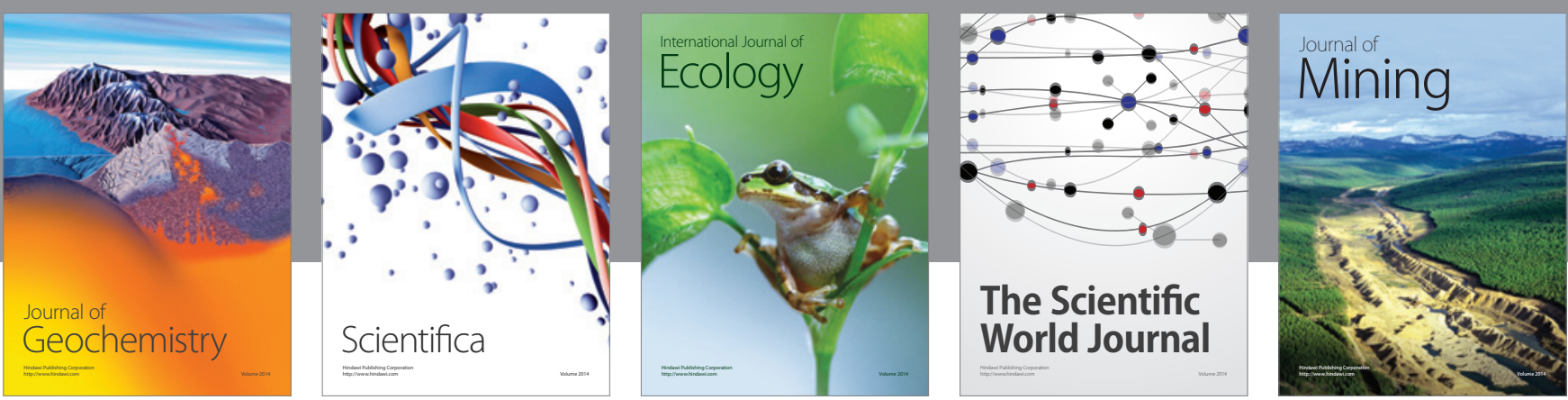

The Scientific World Journal
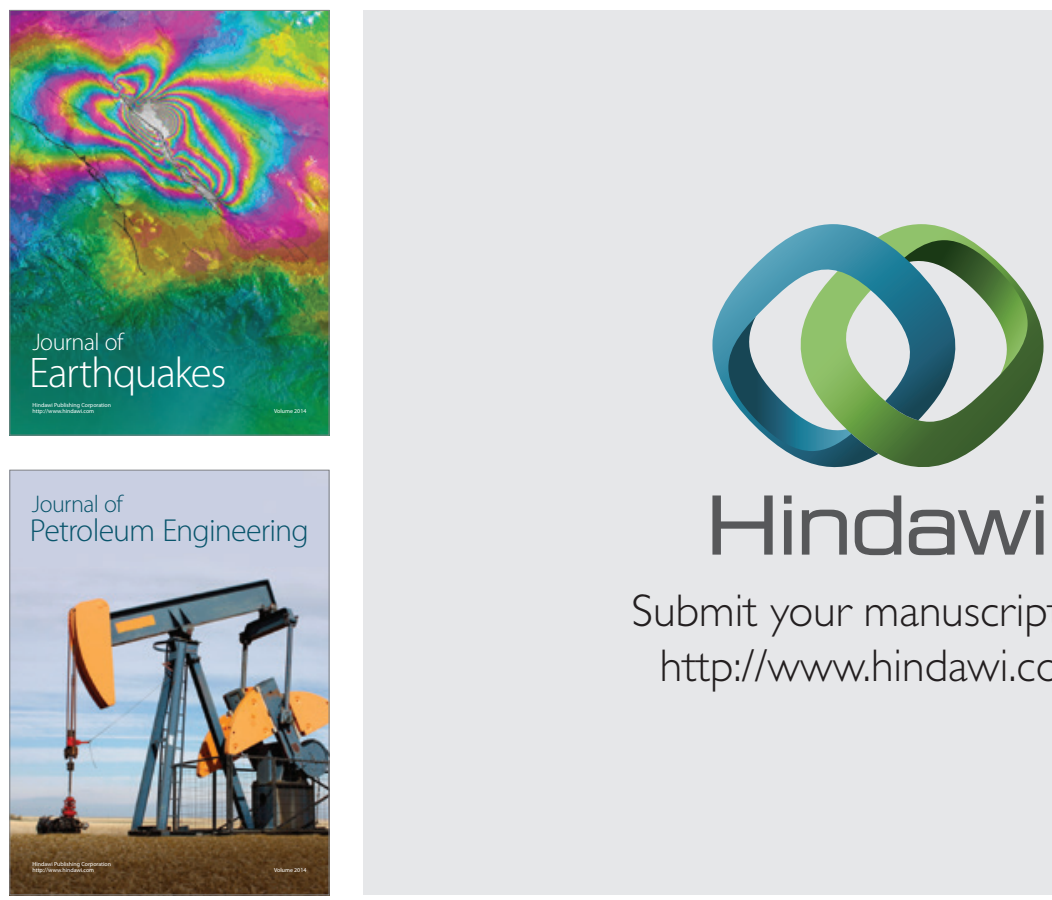

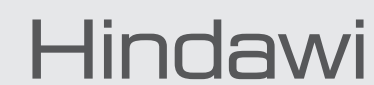

Submit your manuscripts at

http://www.hindawi.com
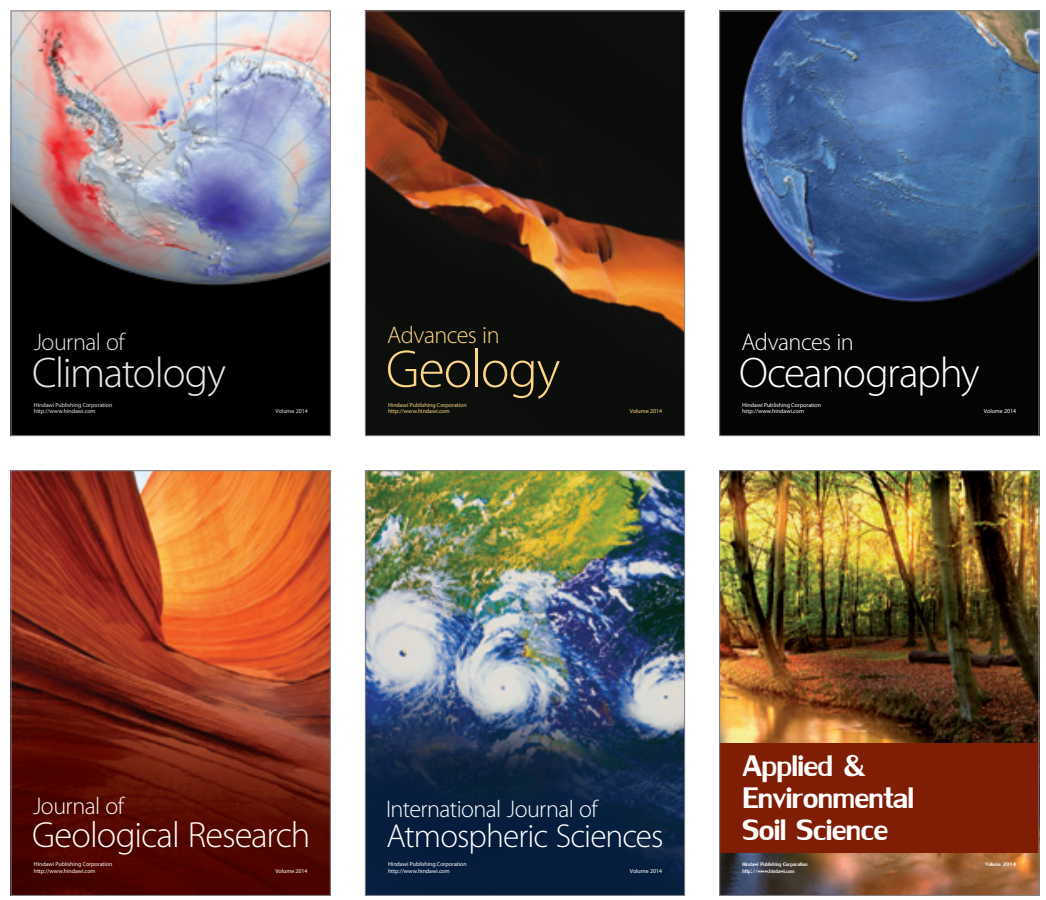
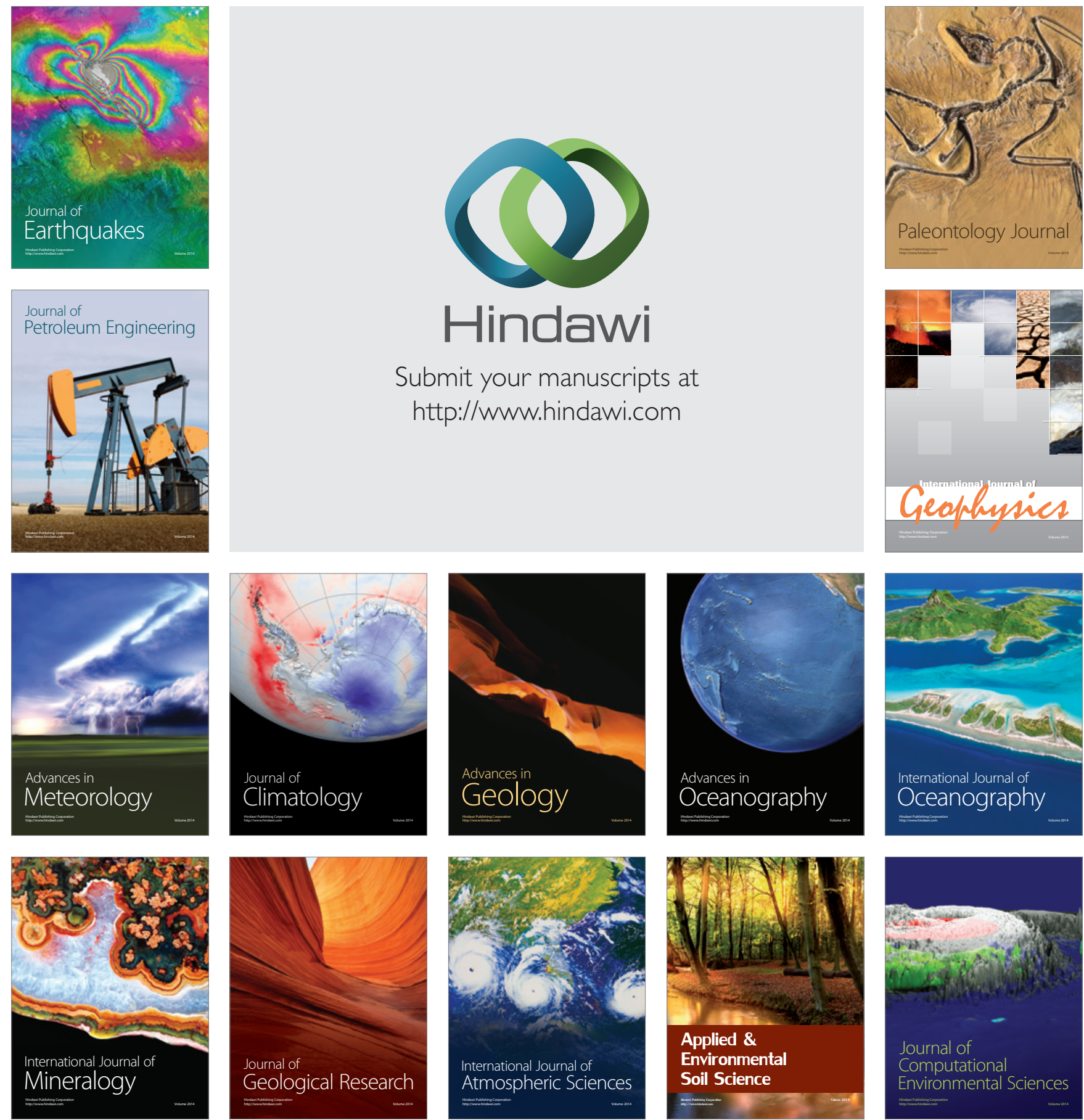